\title{
MicroRNA-375 inhibits glioma cell proliferation and migration by downregulating RWDD3 in vitro
}

\author{
CHEN-XING JI $^{1^{*}}$, YANG-HUA FAN ${ }^{1,2^{*}}$, FAN XU $^{3 *}$, SHI-GANG LV ${ }^{1}$, MIN-HUA YE ${ }^{1}$, \\ MIAO-JING WU ${ }^{1}$, XIN-GEN ZHU ${ }^{1}$ and LEI WU ${ }^{1}$
}

${ }^{1}$ Department of Neurosurgery, The Second Affiliated Hospital of Nanchang University, Nanchang, Jiangxi 330000; ${ }^{2}$ Department of Neurosurgery, Peking Union Medical College Hospital, Chinese Academy of

Medical Sciences/Peking Union Medical College, Beijing 100730; ${ }^{3}$ Department of Neurosurgery,

People's Hospital of Nanchang County, Nanchang, Jiangxi 330200, P.R. China

Received September 2, 2017; Accepted January 31, 2018

DOI: $10.3892 /$ or.2018.6261

Abstract. Derived from brain glial cells, gliomas are currently the most common primary tumours in the central nervous system and are characterised by a high recurrence rate and poor prognosis. RWDD3 (RWD domain-containing sumoylation enhancer, also termed RSUME), which can be induced by cellular stress, such as $\mathrm{CoCl}_{2}$, heat shock and hypoxia, may play a crucial role in tumour angiogenesis, growth and metastasis. MicroRNAs (miRNAs) have been demonstrated to act as negative regulators of post-transcriptional gene expression and are involved in tumour growth and metastasis. In the present study, we explored the role of RWDD3 in glioma cell proliferation and invasion by the knockdown of RWDD3 with lentiviral shRNA and demonstrated that miRNA hsa-miR-375, regulates RWDD3 and has an important role in glioma progression. We found that expression of RWDD3 in high-grade gliomas was significantly higher than that noted in normal brain tissues and lower-grade gliomas in viyo. Knockdown of RWDD3 effectively led to cell cycle arrest, decreased proliferation and invasion, and increased apoptosis in human glioma cell lines. Furthermore, miR-375 was downregulated in human gliomas and overexpression of miR-375 caused downregulation of RWDD3 in glioma cells as well as inhibited their motility. Thus, these findings suggest that RWDD3 and miR-375 may function as therapeutic biomarkers for glioma patients.

orrespondence to: Professor Lei Wu or Professor Xin-Gen Zhu, Department of Neurosurgery, The Second Affiliated Hospital of Nanchang University, 1 Minde Road, Nanchang, Jiangxi 330000, P.R. China

E-mail: robertcarose@163.com

E-mail: zxg2008vip@163.com

*Contributed equally

Key words: RWDD3, miR-375, glioma, proliferation, invasion, migration

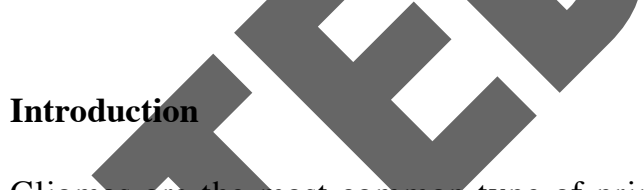

Gliomas are the most common type of primary tumour in the central nervous system (CNS), accounting for $\sim 50 \%$ of all primary nervous system tumours. Despite combined therapy with surgery, radiotherapy and chemotherapy, the poor prognosis and low survival rate of gliomas have been major problems occupying the minds of the medical world (1-3). The median survival time of patients with low-grade gliomas is 6-10 years, while that for patients with high-grade gliomas, such as GBM (World Health Organization grade IV astrocytoma), is only $12-15$ months (4). Therefore, it is necessary to explore the molecular mechanisms underlying glioma occurrence and progression.

RWDD3 (RWD domain-containing sumoylation enhancer, also termed RSUME) was first identified from the lactosomatotrophic tumour cell line GH3, which has higher expression of cytokine transducer gp130 and elevated potential for tumourigenicity and angiogenicity. RWDD3 is highly expressed in various tissues, such as the pituitary, cerebellum and heart, among others (5-8). RWDD3 can also increase I $\kappa$ B levels and, like SUMO, stabilises HIF-1 $\alpha$ during hypoxia, leading to inhibition of NF- $\mathrm{BB}$ and increased HIF-1 transcriptional activity $(8,9)$. Therefore, RWDD3 may play an important role in tumour angiogenesis, growth and metastasis via the HIF-1 $\alpha$ /VEGF pathway. However, its role in glioma is still unclear.

MicroRNAs (miRNAs), a group of small non-coding RNA molecules that are 20-24 nucleotides long, play important roles in specific gene expression. miRNAs act by binding to the 3'-untranslated regions (3'-UTRs) of target gene mRNAs, resulting in mRNA degradation and/or translational repression $(10,11)$. Thus, miRNAs have profound effects on the regulation of biological and pathological processes, such as cell growth, proliferation, apoptosis, metabolism and stress responses $(12,13)$. An abundance of research has shown that the expression patterns of various miRNAs are significantly altered between glioma and normal brain tissue. Various miRNAs are upregulated in gliomas and can promote glioma cell invasion while others have the opposite effect (14-16). 
In the present study, we explored the role of RWDD3 in glioma cell proliferation and invasion by knocking it down with lentiviral shRNA in human glioma cells and demonstrated that miR-375, which is highly expressed in gliomas compared to normal tissues (17), can regulate the expression of RWDD3 and glioma progression.

\section{Patients and methods}

Patients. Glioma tissue samples were collected from 72 patients at the Second Affiliated Hospital of Nanchang University. Patients received no radiotherapy, chemotherapy or immunotherapy before surgery. Brain tissue samples collected from epilepsy surgery were used as controls $(n=10)$. As shown in Table I, the samples were surgically resected from 42 males and 30 females, with an age range of 20 to 62 years old and a mean age of 46.8 years at diagnosis. World Health Organization (WHO) classification criteria were applied for pathological grading of cancer (1). The samples included 12 low-grade astrocytomas (WHO grade I-II), 17 oligodendrocyte astrocytomas (WHO grade II), 13 anaplastic astrocytomas (WHO grade III), and 30 glioblastomas (WHO grade IV). According to the Declaration of Helsinki of 1964 and all subsequent revisions, and with approval of the Ethics Committee of the Second Affiliated Hospital of Nanchang University, the present study was accomplished with informed consent obtained from all patients.

Cell culture. U251 and U87 human glioma cell lines and human glial cell line HEB were maintained in our laboratories. These cells were cultured in Dulbecco's modified Eagle's medium (DMEM) containing 10\% foetal bovine serum(FBS) (both from Bioind, Kibbutz Beit Haemek, Israel) at $37^{\circ} \mathrm{C}$ with $5 \% \mathrm{CO}_{2}$.

Cell transfection. Mimics of miR-375 and negative controls were purchased from RiboBio Company (Guangzhou, China). The pcDNA/RWDD3 and control vectors were purchased from GenePharma Company (Shanghai, China), Transfection was performed using Lipofectamine ${ }^{\mathrm{TM}} 2000$ (Invitrogen; Thermo Fisher Scientific, Inc., Waltham, MA, USA) according to the manufacturer's protocol. Cells were harvested for ensuing experiments $48 \mathrm{~h}$ after transfection. Lentiviral GV248 vector and a package system, Lentivector Packaging kit, were purchased from Shanghai Jikai, Inc. (Shanghai, China). The RWDD3-shRNA plasmid was constructed by inserting the targeting sequence 5'-GATGATGGATTGTGGATAA-3' into the GV248 vector. Lentiviral particles were produced using the Lentivector Packaging kit and transduced into cells according to the manufacturer's protocol (Shanghai Jikai, Inc.). Blank and empty vector control groups (NC-shRNA) were included as controls for the RWDD3-shRNA experimental group.

Western blot analysis. Tissue homogenate or cultured cells were lysed with a hypotonic buffer containing $2 \%$ Nonidet P-40 and a protease inhibitor cocktail (Sigma-Aldrich; Merck KGaA, Darmstadt, Germany) by sonication three times for $3 \mathrm{sec}$ on ice. The supernatant obtained after centrifugation at $2,000 \mathrm{x} \mathrm{g}$ for $15 \mathrm{~min}$ at $4^{\circ} \mathrm{C}$ was used for all subsequent steps, and the protein concentration was determined by the Coomassie blue
Table I. Characteristics of the glioma patients $(n=72)$.

\begin{tabular}{|c|c|c|}
\hline \multirow[b]{2}{*}{ Characteristics } & \multicolumn{2}{|c|}{ No. of cases } \\
\hline & $\mathrm{N}$ & $(\%)$ \\
\hline \multicolumn{3}{|l|}{ Sex } \\
\hline Male & 42 & $(58.3)$ \\
\hline Female & 30 & $(41.7)$ \\
\hline \multicolumn{3}{|l|}{ Age (years) } \\
\hline$\leq 50$ & 43 & $(59.7)$ \\
\hline$>50$ & 29 & $(40.3)$ \\
\hline \multicolumn{3}{|l|}{ Body mass index } \\
\hline$<20$ & 5 & $(20.8)$ \\
\hline $20-28$ & & $(66.7)$ \\
\hline$>28$ & & $(12.5)$ \\
\hline \multicolumn{3}{|l|}{ Family history } \\
\hline Positive & & (15.3) \\
\hline N & 61 & $(84.7)$ \\
\hline & 48 & \\
\hline & 24 & (33.3) \\
\hline & 45 & $(62.5)$ \\
\hline & 27 & $(37.5)$ \\
\hline & 29 & $(40.3)$ \\
\hline $\mathrm{III} / \mathrm{I}$ & 43 & $(59.7)$ \\
\hline
\end{tabular}

WHO, World Health Organization.

method. Equal amounts of proteins for each sample were separated on $8-15 \%$ SDS-polyacrylamide gels and blotted onto polyvinylidene difluoride microporous membranes (Millipore, Billerica, MA, USA). Membranes were blocked with 5\% skim milk powder in TBS-T for $2 \mathrm{~h}$ and then incubated for $1 \mathrm{~h}$ with a 1:800 dilution of rabbit polyclonal anti-human RWDD3 (ab128285) antibody or a 1:5,000 dilution of rabbit polyclonal anti-human $\beta$-actin (ab189073) antibody (Abcam, Cambridge, UK). Membranes were then washed and incubated with bovine anti-rabbit (sc-237; Santa Cruz Biotechnology, Santa Cruz, CA, USA) secondary antibody $(1: 5,000,1 \mathrm{~h})$. Peroxidase signals were detected with a GE Healthcare ECL kit (Shanghai, China). Three independent experiments were performed.

Quantitative real-time polymerase chain reaction ( $R T-q P C R)$ assay. Total RNA was extracted from glioma tumour tissue, adjacent normal tissue, and glioma cell lines using the TRIzol Total RNA Reagent (Invitrogen; Thermo Fisher Scientific, Inc.). Complementary DNA (cDNA) synthesis was performed with $2 \mu \mathrm{g}$ of total RNA using the PrimeScript ${ }^{\mathrm{TM}}$ RT reagent kit (RR037A; Takara, Otsu, Shiga, Japan). Primers for miR-375 and RNU6B (U6 small nuclear B non-coding RNA) were synthesised and purchased from RiboBio Company. Primers for RWDD3 and GAPDH were synthesised and purchased from GenScript Company (Nanjing, China). The RWDD3 primer 

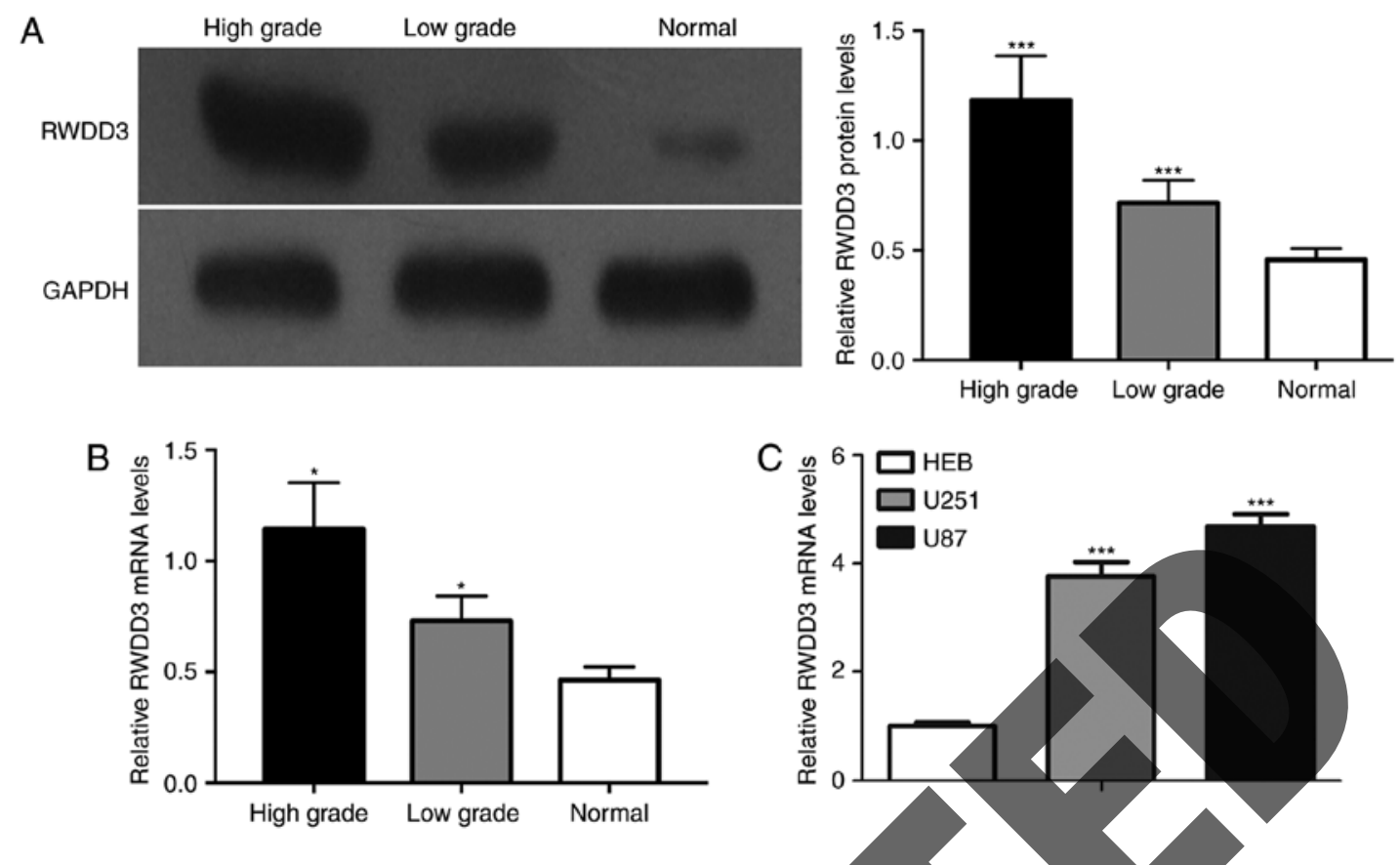

Figure 1. Significant differences in RWDD3 expression in vivo and in vitro (A) The expression of RWDD3 proteins in different grade gliomas and normal brain tissues was detected by western blotting. GAPDH was used as an internal control. (B) The expression of RWDD3 mRNAs in different grade gliomas and normal brain tissues was detected by RT-qPCR. (C) The expression of RWDD3 mRNAs in glioma cells and human glial cell line HEB was detected by real-time qPCR. ${ }^{\mathrm{P}}<0.05,{ }^{* * *} \mathrm{P}<0.001$. RWDD3, RWD domain-containing sumoylation enhancer.

sequences were: forward primer, 5'-TACCTGGTATCTCGA was calculated with ImageJ software (National Institutes of TTA-ACTCTGAAC-3' and reverse primer, 5'-TCAGTATTA Health, Bethesda, MD, USA).

TTTTACCCATGAACATCA-3'; the GAPDH primer sequences were: forward primer, 5'-GTTGGAGGTCGGAGTCAA Cell Counting Kit-8 assay. Transfected U251 and U87 cells CGG-3' and reverse primer, 5'-GAGGGATCTCGCTCCTGG were seeded in 96-well plates $\left(2 \times 10^{3}\right.$ cells/well) and cultured AGGA-3'. RT-qPCR was performed using the SYBR ${ }^{\circledR}$ Premix for 24, 48, 72, 96 and $120 \mathrm{~h}$. Thereafter, the cells were incuEx Taq ${ }^{\mathrm{TM}}$ (RR420A; Takara) using the Applied Biosystems 7300 Fluorescent Quantitative PCR System (Thermo Fisher Scientific, Inc.). Reaction mixtures were incubated at $95^{\circ} \mathrm{C}$ for $30 \mathrm{sec}$, followed by 40 amplification cycles of $95^{\circ} \mathrm{C}$ for $5 \mathrm{sec}$ and $60^{\circ} \mathrm{C}$ for $34 \mathrm{sec}$. Gene expression was quantified using the $\Delta \Delta \mathrm{CT}$ calculation where $\mathrm{CT}$ was the threshold cyele.

Transwell assay. Transwell chambers (Corning, Corning, NY, USA) were utilised to explore invasion and migration abilities. For the migration assay, transfected cells were inoculated in DMEM with serum-free medium $\left(5 \times 10^{4}\right.$ cells $)$ in the upper chamber while the lower chamber contained DMEM with $10 \%$ FBS. For invasion assays, the chamber was covered with Matrigel (10 mg/ml; BD Biosciences, Bedford, MA, USA). After $24 \mathrm{~h}$ of cell migration and invasion, cells were washed with phosphate solution, fixed with polyformaldehyde and stained with $0.1 \%$ crystal violet. Treated cells were photographed under a microscope and counted.

Scratch assay. U251 and U87 cells were seeded in 6-well plates $\left(2 \times 10^{5}\right.$ cells/well). At $48 \mathrm{~h}$ after transfection ( $90 \%$ confluence), a linear wound area was marked with a sterile $10 \mu 1$ pipette tip, washed with phosphate solution to remove detached cells, and supplemented with fresh DMEM without FBS. Cells were then incubated at $37^{\circ} \mathrm{C}$ and $5 \% \mathrm{CO}_{2}$. The width of the wound area was surveyed with an inverted microscope at different time-points and the normalised wound area bated with $10 \mu \mathrm{l} /$ well of Cell Counting Kit-8 (CCK-8) solution (TransGen, Beijing, China) for $2 \mathrm{~h}$ at $37^{\circ} \mathrm{C}$. The absorbance of the samples at $450 \mathrm{~nm}$ was detected with a Varioskan LUX Multimode Microplate Reader (Thermo Fisher Scientific, Inc.).

Apoptosis analysis. Cell apoptosis was detected with an APC Annexin V kit with a FACSCalibur flow cytometry system according to the manufacturer's protocol (BD Biosciences, Bedford, MA, USA).

Statistical analysis. The IBM SPSS 20.0 statistical software (IBM Corp., Armonk, NY, USA) was utilised to calculate and analyse our experimental data. Experiments were repeated three times, and the data are presented as the means \pm standard deviations (SD). All data were statistically analysed using a one-way analysis of variance with a Bonferroni correction while bivariate correlations were calculated based on Spearman's rank correlation coefficients. Differences with $\mathrm{P}$-value $<0.05$ were considered statistically significant.

\section{Results}

Significant differences in RWDD3 expression in vivo and in vitro. Western blot and RT-qPCR assays showed that protein (Fig. 1A) and mRNA (Fig. 1B) levels of RWDD3 in high-grade gliomas were significantly higher than those in normal brain tissues and lower-grade gliomas. As shown 


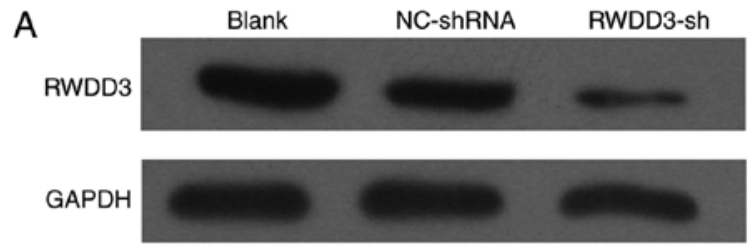

U251
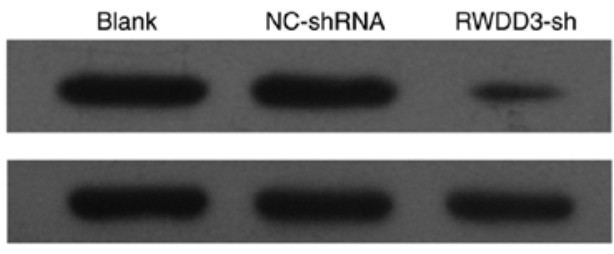

U87

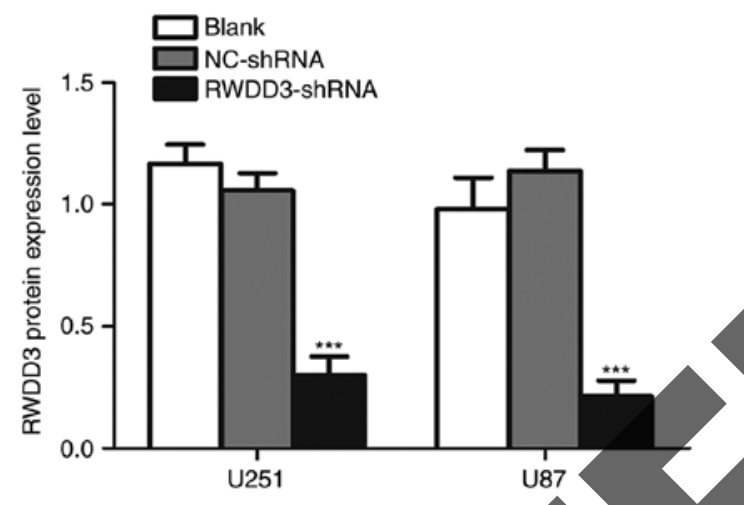

B

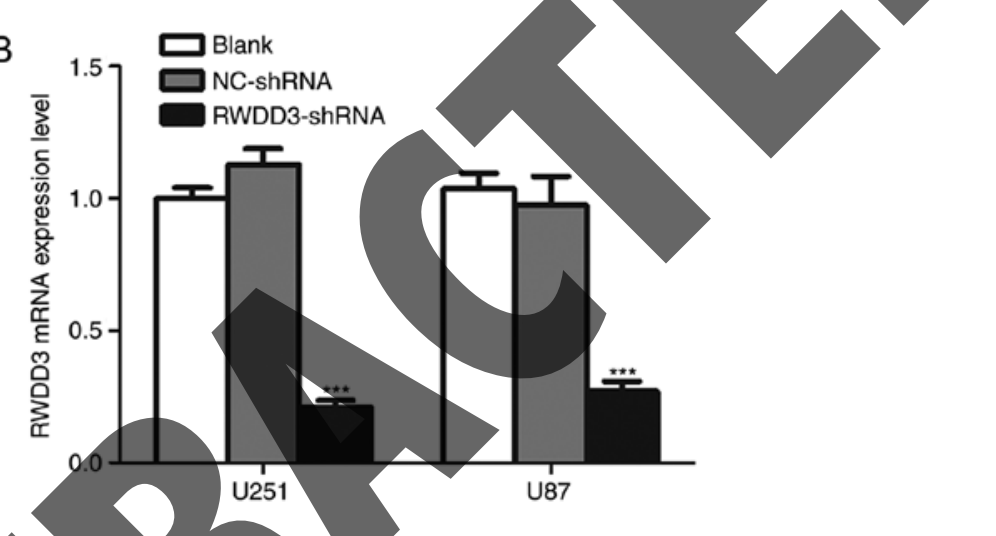

Figure 2. (A) RWDD3-shRNA effectively knocks down RWDD3 protein and mRNA levels in glioma cells. The expression of RWDD3 protein in glioma cells was detected by western blotting after transfection with RWDD3-shRNA and its control. GAPDH was used as an internal control. (B) The expression of RWDD3 mRNA in glioma cells was detected by RT-qPCR after transfection with RWDD3-shRNA and its controls. ${ }^{* * *} \mathrm{P}<0.001$. RWDD3, RWD domaincontaining sumoylation enhancer.

in Fig. 1C, the mRNA levels of RWDD3 in the U251 and U87 glioma cells were higher than that in the normal human glial cell line, HEB.

Knockdown of RWDD3 by lentivirah shRNA effectively led to cell cycle arrest, decreased proliferation and invasion, and increased apoptosis. We next transduced the U251 and U87 cells with lentiviral RWDD3-shRNA. As shown in Fig. 2A, RWDD3-shRNA knocked down the RWDD3 protein level by $\sim 70 \%$ in both cell lines compared with the controls. As shown in Fig. 2B, RWDD3-shRNA knocked down the RWDD3 mRNA level by $\sim 80 \%$ in both cell lines relative to the blank and empty lentiviral vector controls.

CCK-8 proliferation assays showed significantly decreased proliferation of U251 and U87 cells transduced with RWDD3-shRNA compared to the blank and empty lentiviral vector controls (Fig. 3A). Transwell cell invasion assays showed an $\sim 40 \%$ decrease in cell invasiveness in the U251 and U87 cells transduced with RWDD3-shRNA compared with the controls (Fig. 3B).

Flow cytometric analyses showed that in both U251 and U87 cells, knockdown of RWDD3 by lentiviral shRNA increased the percentage of cells at the G0/G1 cell cycle phase by over $20 \%$ compared with the controls (Fig. 3C). As shown in Fig. 3D, knockdown of RWDD3 by lentiviral shRNA increased cell apoptosis by $\sim 4 \%$ in $\mathrm{U} 251$ cells and by $\sim 7 \%$ in U87 cells relative to the controls.

miR-375 overexpression inhibits glioma cell proliferation and migration. After transfection with miR-375 mimics and controls into U251 and U87 cells, we investigated the miR-375 levels and the resulting functional differences. The expression of miR-375 was significantly higher in cells transduced with the miR-375 mimics compared with that noted in the controls (Fig. 5C). Based on the CCK-8 proliferation assay, we found that miR-375 overexpression inhibited U251 and U87 cell proliferation compared with the controls (Fig. 4A). Furthermore, we tested the migration of the transfected glioma cells by Transwell assays. As shown in Fig. 4B, the migration capability of the U251 and U87 cells was significantly reduced by the miR-375 mimics but not by the controls. Consistent with the Transwell migration results, scratch assays showed that the migration capability of the U251 and U87 cells was also inhibited by the miR-375 mimics (Fig. 4C). All the results above 

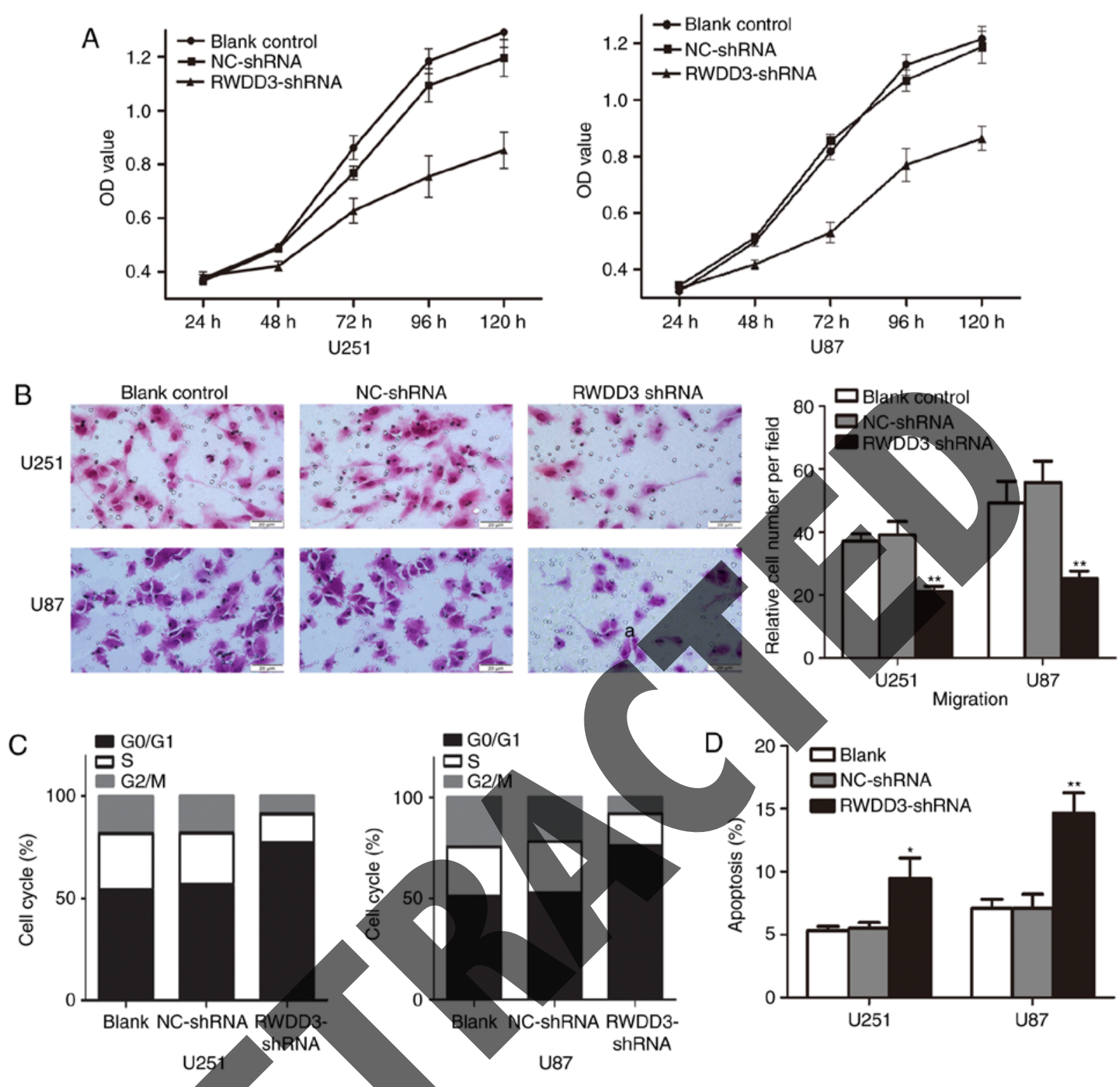

Figure 3. Knockdown of RWDD3 effectively leads to decreased proliferation and invasion, cell cycle arrest and increased apoptosis in human glioma cell lines. (A) CCK-8 proliferation assays were used to detect cell proliferation after transfection with RWDD3-shRNA and its controls. (B) To test the invasion of transfected cells, Transwell chambers coated with Matrigel were used. (C) Cell cycle progression was detected by flow cytometric analyses. (D) Cell apoptosis was detected by flow cytometric analyses. Values are represented as mean $\pm \mathrm{SD}$ of three separate experiments. ${ }^{*} \mathrm{P}<0.05,{ }^{* *} \mathrm{P}<0.01$. RWDD3, RWD domaincontaining sumoylation enhancer.

suggest that miR-375 acts as an important negative regulator of glioma metastasis.

miR-375 inhibits RWDD3 expression. To determine whether RWDD3 is regulated by miR-375, we performed western blot analyses and RT-qPCR in glioma cells transfected with miR-375 mimics or in negative control cells. As shown in Fig. 5A and B, overexpression of miR-375 clearly restrained the expression of RWDD3 at both the protein and mRNA levels in comparison with the negative control.

Overexpression of $R W W D 3$ rescues cell proliferation and migration in U251 and U87 cells inhibited by miR-375. To clarify the mechanism of miR-375 in inhibiting cell proliferation and migration through regulation of RWDD3, we performed a series of rescue experiments by means of simultaneous transfection with the miR-375 mimics and pcDNA/RWDD3 into glioma cells. As shown in Fig. 6A-C, RWDD3 clearly enhanced the cell proliferation and migration abilities that had been suppressed by the miR-375 mimics.

Overexpression of RWWD3 restores the low RWDD3 levels reduced by $m i R-375$. After ascertaining that the expression of RWWD3 protein and mRNA were inhibited by miR-375 mimics, we performed co-transfection with the miR-375 mimics and pcDNA/RWDD3 into the U251 and U87 cells. As shown in Fig. 7A and B, overexpression of RWDD3 restored the low RWDD3 levels observed by transfection of miR-375 alone.

All these experimental results suggest that RWDD3 functions as an important inductor in glioma metastasis, while overexpression of miR-375 inhibits cell proliferation and migration in U251 and U87 cells by reducing RWDD3 expression. 

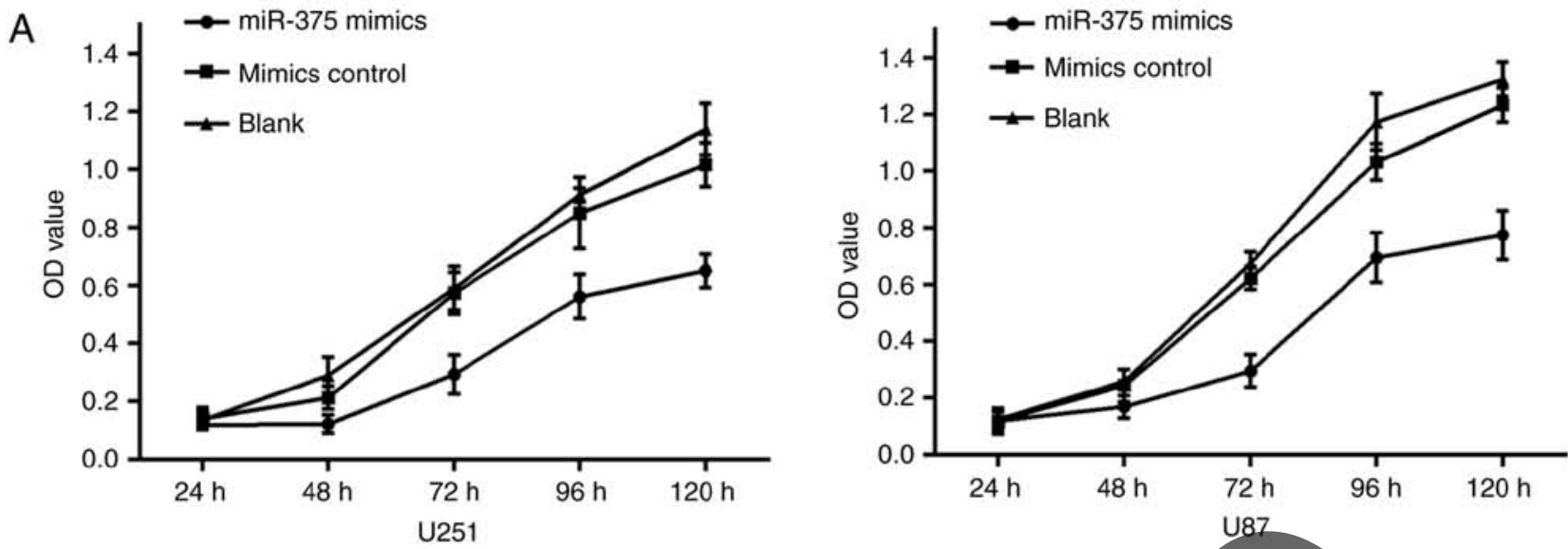

B
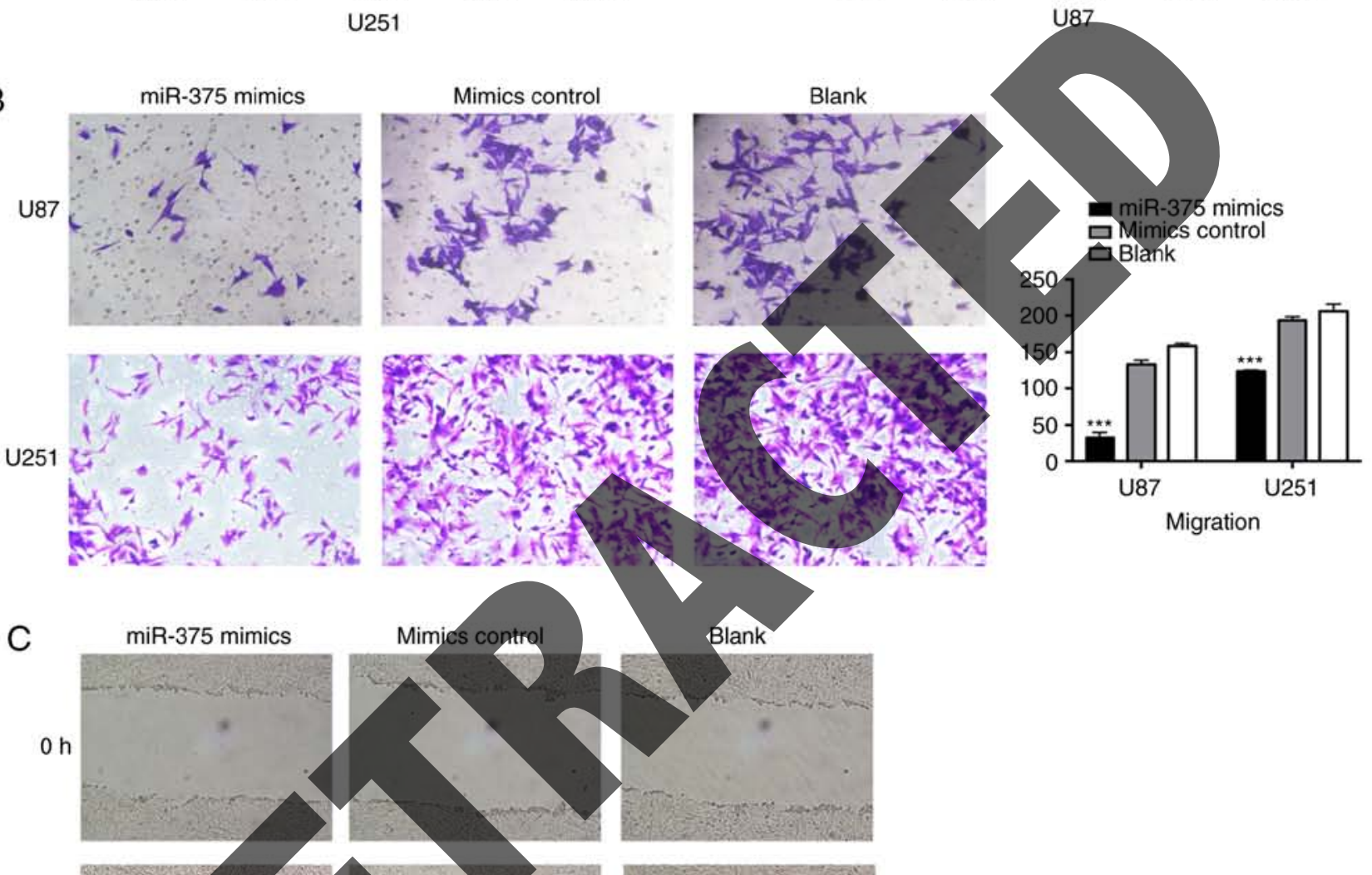

$48 \mathrm{~h}$
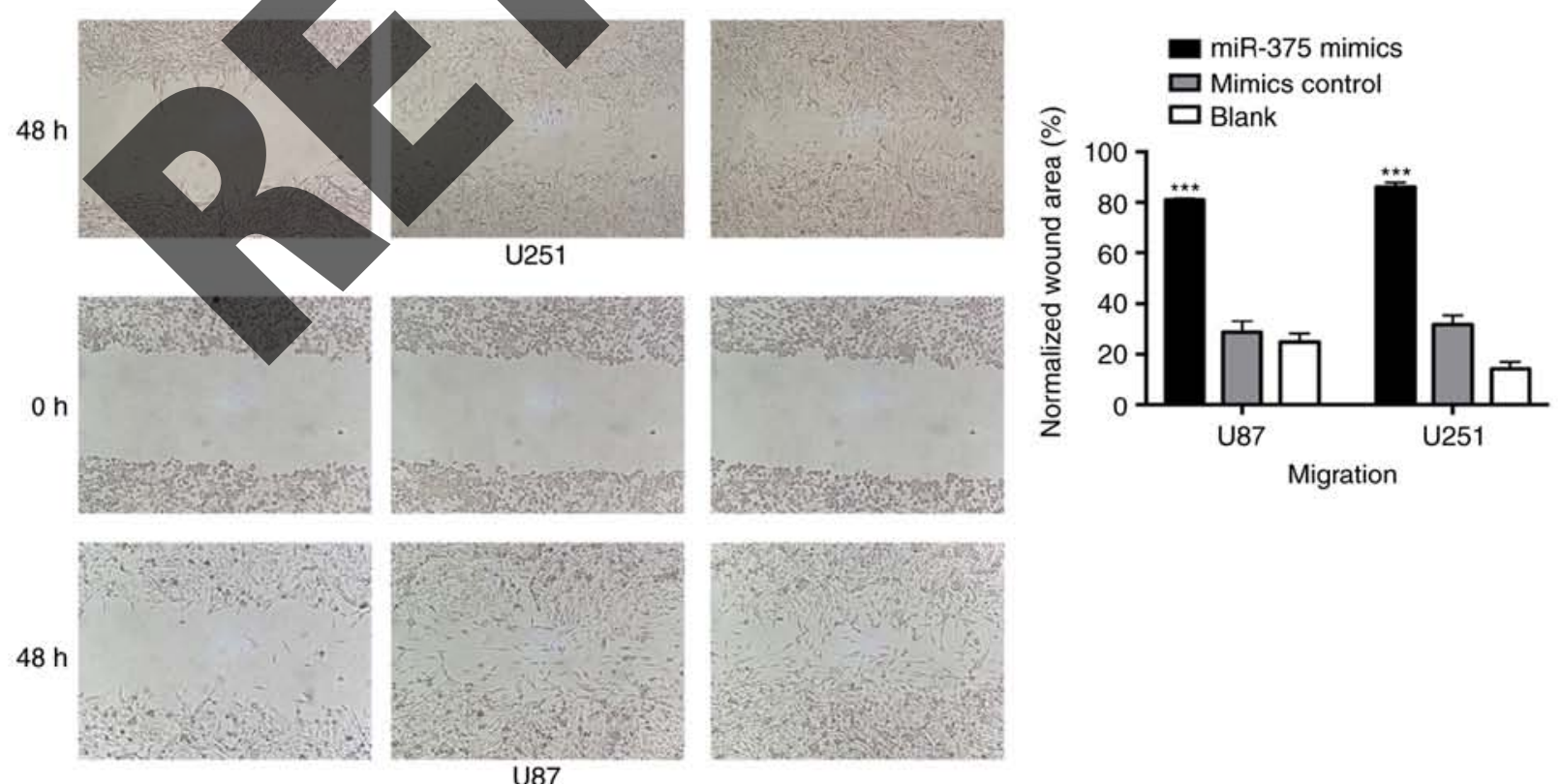

Figure 4. (A) Overexpression of miR-375 inhibits the proliferation and migration of glioma cells. (B) CCK-8 proliferation assays were used to detect cell proliferation after transfection with miR-375 mimics and controls. (C) Transwell assays were used to investigate cell invasion. Scratch assays were used to investigate cell migration. Values are represented as mean \pm SD of three separate experiments. ${ }^{* * *} \mathrm{P}<0.001$. 
A

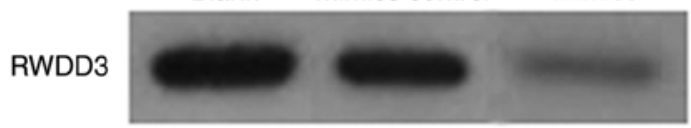

GAPDH

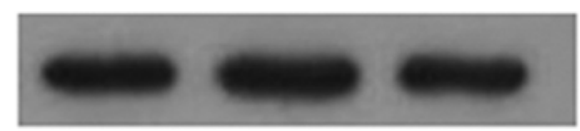

U251

B
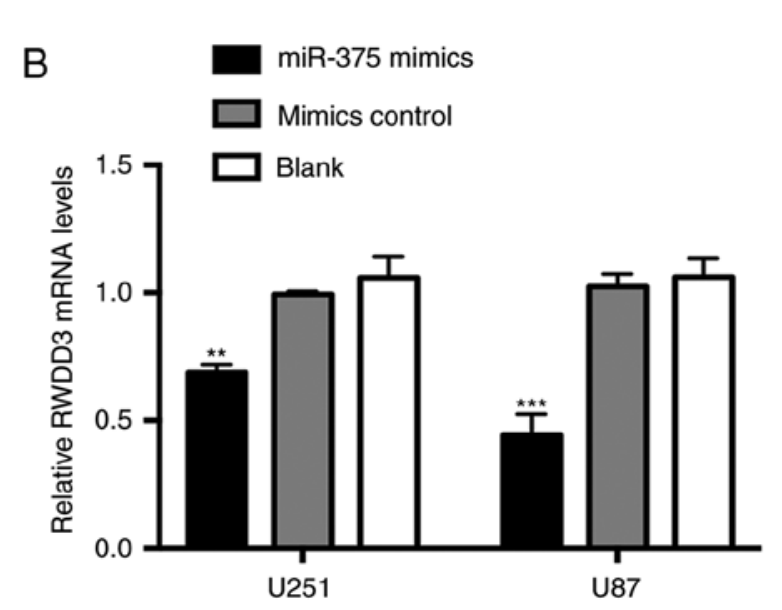
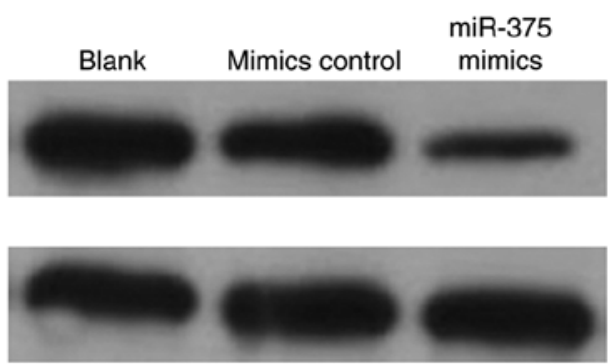

U87

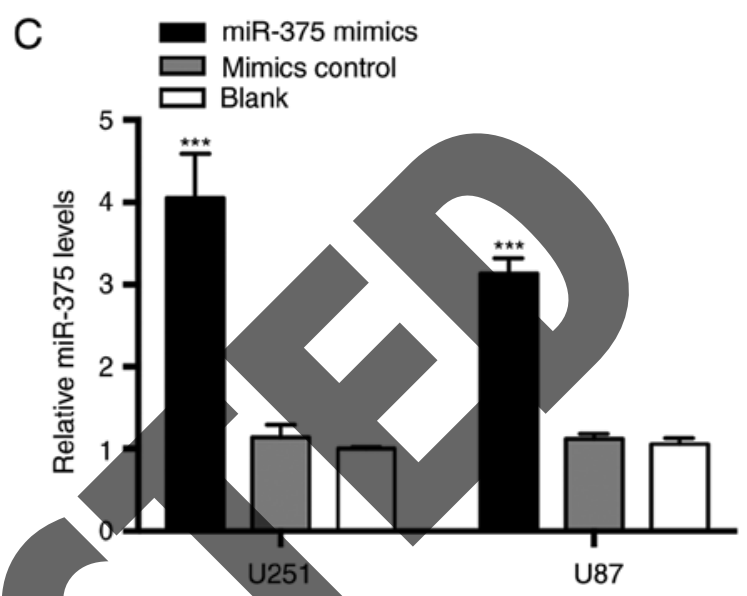

Figure 5. Expression levels of RWDD3 and miR-375 in glioma cells after transfection with miR-375 mimics and controls were detected by western blotting and RT-qPCR. (A) The expression of RWDD3 proteins in glioma cells was detected by western blotting after transfection with miR-375 mimics and controls. GAPDH was used as an internal control. (B) The expression of RWDD3 mRNA in glioma cells was detected by RT-qPCR after transfection with miR-375 mimics and its controls. (C) The levels of miR-375 were detected by RT-qPCR after transfection with miR-375 mimics and controls. U6 was used as an internal control. ${ }^{* *} \mathrm{P}<0.01,{ }^{* * * *} \mathrm{P}<0.001$. RWDD3, RWD domain-containing sumoylatiòn enhancer

\section{Discussion}

Patients with gliomas, particularly ghioblastomas, have generally poor prognoses (1-3). Gliomas are some of the most common highly vascularised malignant tumours in the CNS, with a $\leq 12$-month median patient survival period (4). Despite advances in diagnosis and treatment, frequent recurrence and low cure rates of gliomas remain an urgent issue due to the rapid proliferation and high invasiveness of this disease (4).

Rapid tumour growth is often accompanied by lack of tumour blood supply, which normally produces a hypoxic microenvironment $(18,19)$. To maintain the tumour blood supply, the ability of tumour blood vessels to survive increases. Previous studies have found that the HIF-1 $\alpha /$ VEGF pathway plays an important role in this process in gliomas $(18,19)$. HIF-1 is composed of a heterologous dimer of HIF-1 $\alpha(120 \mathrm{kDa})$ and HIF-1 $\beta$ (91-94 kDa) subunits, in which HIF-1 $\alpha$ is the major active unit (18). Hypoxia induces expression of HIF-1 $\alpha$, which regulates the expression of many genes involved in tumour cell proliferation and invasion (19). However, under normoxia, HIF-1 $\alpha$ undergoes rapid oxygen-dependent ubiquitination at Lys391 and Lys477, which leads to its subsequent degradation (6). The small ubiquitin related modifier family has three members: SUMO-1, SUMO-2, and SUMO-3. With a similar structure to that of ubiquitin, SUMO-1 can compete with ubiquitin for HIF-1 $\alpha$ modification and thus protect HIF-1 $\alpha$ from degradation (20-24).

Originally identified in the lactosomatotrophic tumour cell line GH3, which overexpresses the cytokine transducer gp130 and has increased tumourigenic and angiogenic potential, RWDD3 can be induced by cellular stress, such as $\mathrm{CoCl}_{2}$, heat shock and hypoxia, and its expression is also increased in gliomas $(5-10,18)$. RWDD3 has higher expression in various tissues, such as the pituitary, cerebellum, heart, liver, kidney, pancreas, adrenal gland and prostate (5). Recent studies have shown that RWDD3 interacts with Ubc9, the SUMO conjugase, to increase overall SUMO-1, -2 and -3 conjugation. RWDD3 increases non-covalent binding of SUMO-1 to Ubc9, enhances Ubc9 thioester formation and SUMO polymerization. In addition, its expression is induced by hypoxia and it enhances the sumoylation of HIF- $1 \alpha$, promoting its stability and transcriptional activity during hypoxia. Thus, by stabilizing HIF-1 $\alpha$, RWDD3 may play an important role in tumour angiogenesis, growth and metastasis $(5-8,18)$.

MicroRNAs (miRNAs), a group of small non-coding RNA molecules with a length of $20-24$ nucleotides, play important roles in gene expression. The 3'-untranslated regions (3'-UTRs) of target gene mRNAs can be modified by corresponding miRNAs which results in mRNA degradation and/or translational repression $(10,11)$. Although each miRNA has multiple target genes, we used bioinformatic methods to predict target miRNAs that may regulate RWDD3. Based on results from TargetScan, miRanda and StarBase, we selected miR-375 as a target for further validation. Previous studies have shown that miR-375 is tightly associated with the occurrence and development of tumours. Wei et al found that miR-375 inhibits the proliferation of colorectal cancer cells by downregulating JAK2/STAT3 and MAP3K8/ERK signalling 

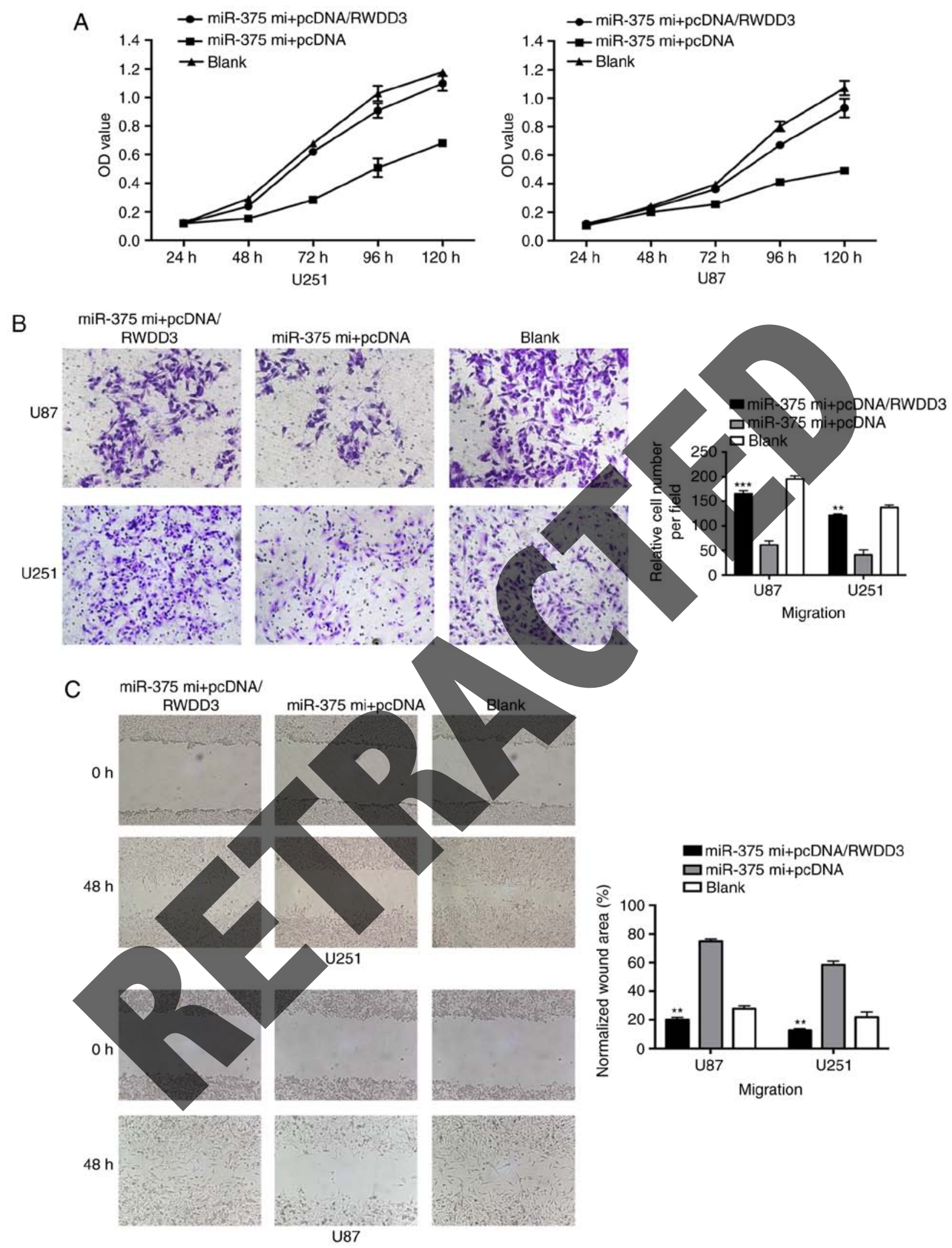

Figure 6. Overexpression of RWDD3 enhances glioma cell proliferation and migration reduced by miR-375. (A) CCK-8 proliferation assays were used to detect cell proliferation after co-transfection with miR-375 mimics and pcDNA/RWDD3. (B) Transwell assays were used to investigate migration. (C) Scratch assays were used to investigate migration. Values are represented as mean $\pm \mathrm{SD}$ of three separate experiments. ${ }^{* * *} \mathrm{P}<0.001$. RWDD3, RWD domain-containing sumoylation enhancer.

pathways (25). Tsukamoto et al demonstrated that miR-375 is downregulated in gastric carcinomas and regulates cell survival by targeting PDK1 and 14-3-3zeta (26). Chang et al showed that on average, miR-375 expression was significantly 


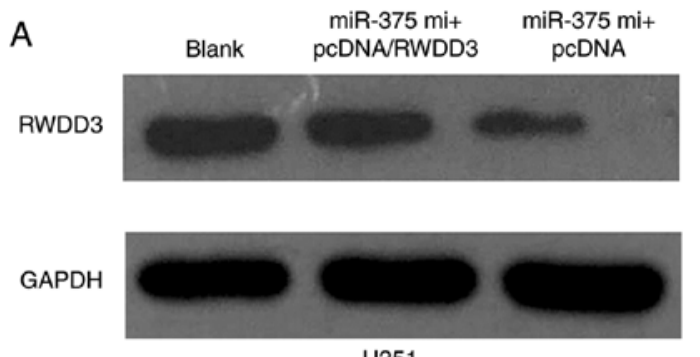

U251
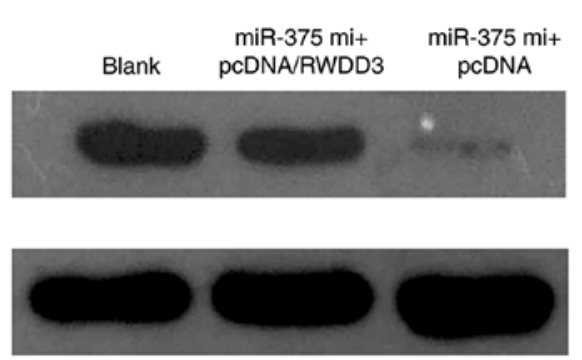

U87

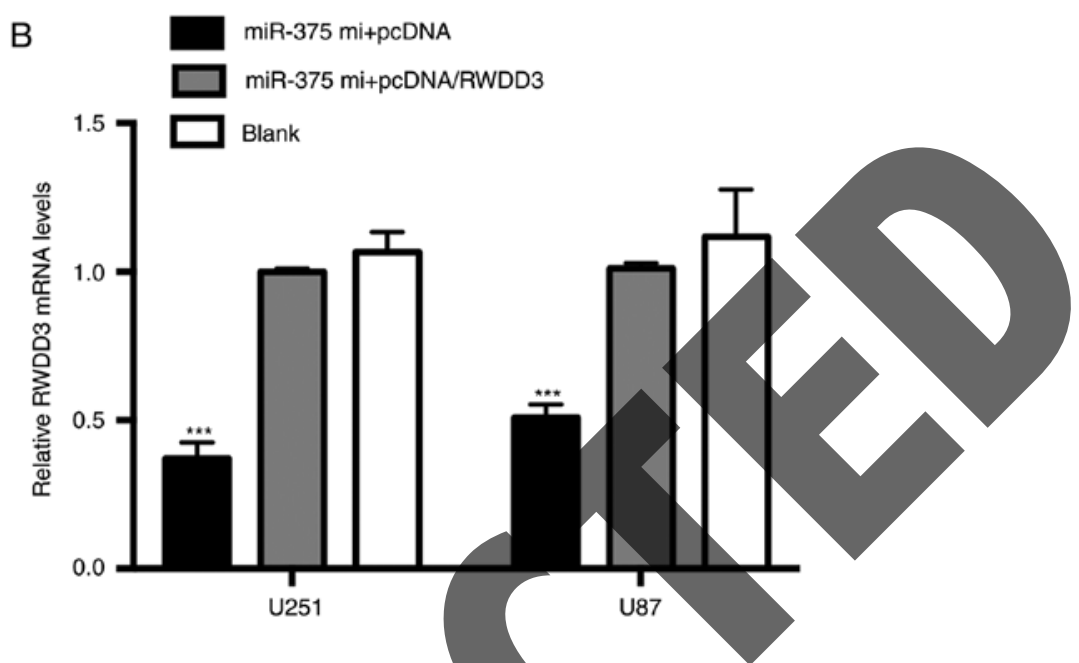

Figure 7. Overexpression of RWDD3 restores RWDD3 levels reduced by miR-375. (A) The expression of RWDD3 proteins in glioma cells was detected by western blotting after co-transfection with miR-375 mimics and pcDNA/RWDD3. GAPDH was used as an internal control. (B) The expression of RWDD3 mRNA in glioma cells was detected by RT-qPCR after transfection with miR-375 mimics and pcDNA/RWDD3. *** P<0.001. RWDD3, RWD domain-containing sumoylation enhancer.

decreased in glioma tissues relative to non-neoplastic brain Availability of data and materials tissues with ascending pathological grade (17).

In the present study, we found that RWDD3 was expressed at significantly higher levels in high-grade gliomas than in low-grade gliomas and normal brain tissues in vivo. Our in vitro data showed that knockdown of RWDD3 effectively led to cell cycle arrest, decreased proliferation and invasion, and increased apoptosis in human glioma cell lines. Overexpression of miR-375 effectively inhibited glioma cell proliferation and migration, while RWDD3 overexpression restored cell proliferation/and migration after overexpression of miR-375. Thus, miR-375 and RWDD3 could be new potential therapeutic targets for glioma.

\section{Acknowledgements}

This manuscript was edited for English language by Elsevier.

\section{Funding}

The present study was supported by the National Natural Science Foundation of China (no. 81560411), the Construction Plan of the Superior Science and Technology Innovation Team of Jiangxi Province (no. 20152BCB24009), the Foreign Science and Technology Cooperation Plan of Jiangxi Province (no. 20151BDH80009), the Jiangxi Province's Department of Education Science and Technology Research Project (no. GJJ160253), and the Innovation Fund for graduates of Nanchang University (no. cx2016327).

The datasets used and/or analyzed during the current study are available from the corresponding author on reasonable request.

\section{Authors' contributions}

CXJ performed the cell culture, cell transfection, the Cell Counting Kit-8 assay and apoptosis analysis and was a major contributor in writing the manuscript. YHF performed the western blot analysis and RT-qPCR analysis. FX performed the Transwell assay and scratch assay. SGL collected and analyzed the patient data. MHY contributed to acquisition of data. MJW performed the statistical analysis. XGZ contributed to conception and design. LW contributed to conception and design and revised it critically for important intellectual content. All authors read and approved the final manuscript.

\section{Ethics approval and consent to participate}

According to the Declaration of Helsinki of 1964 and all subsequent revisions, and with approval of the Ethics Committee of the Second Affiliated Hospital of Nanchang University, the present study was accomplished with informed consent obtained from all patients.

\section{Consent for publication}

Not applicable. 


\section{Competing interests}

The authors declare that they have no competing interests.

\section{References}

1. Rainov NG and Heidecke V: Clinical development of experimental therapies for malignant glioma. Sultan Qaboos Univ Med J 11: 5-28, 2011 .

2. Dong J, Wang XQ, Yao JJ, Li G and Li XG: Decreased CUL4B expression inhibits malignant proliferation of glioma in vitro and in vivo. Eur Rev Med Pharmacol Sci 19: 1013-1021, 2015.

3. Norden AD, Drappatz J and Wen PY: Antiangiogenic therapies for high-grade glioma. Nat Rev Neurol 5: 610-620, 2009.

4. Castro MG, Candolfi M, Kroeger K, King GD, Curtin JF, Yagiz K Mineharu Y, Assi H, Wibowo M, Ghulam Muhammad AK, et al: Gene therapy and targeted toxins for glioma. Curr Gene Ther 11: 155-180, 2011.

5. Fuertes M, Gerez J, Haedo M, Giacomini D, Páez-Pereda M, Labeur M, Stalla GK and Arzt E: Cytokines and genes in pituitary tumorigenesis: RSUME role in cell biology. Front Horm Res 38 $1-6,2010$.

6. Carbia-Nagashima A, Gerez J, Perez-Castro C, Paez-Pereda M, Silberstein S, Stalla GK, Holsboer F and Arzt E: RSUME, a small RWD-containing protein, enhances SUMO conjugation and stabilizes HIF-1alpha during hypoxia. Cell 131: 309-323, 2007.

7. Fowkes RC and Vlotides G: Hypoxia-induced VEGF production 'RSUMEs' in pituitary adenomas. Endocr Relat Cancer 19: C1-C5, 2012.

8. Gerez J, Fuertes M, Tedesco L, Silberstein S, Sevlever G, Paez-Pereda M, Holsboer F, Turjanski AG and Arzt E: In silico structural and functional characterizations of the RSUME splice variants. PLoS One 8: e57795, 2013.

9. Chan JY, Tsai CY, Wu CH, Li FC, Dai KY, Sun EY, Chan SH and Chang AY: Sumoylation of hypoxia-inducible factor-1 $\alpha$ ameliorates failure of brain stem cardiovascular regulation in experimental brain death. PLoS One 6: e17375, 2011.

10. Bartel DP: MicroRNAs: Genomics, biogenesis, mechanism, and function. Cell 116: 281-297, 2004.

11. Shukla GC, Singh J and Barik S: MicroRNAs: Processing, maturation, target recognition and regulatory functions. Mol Cell

Pharmacol 3: 83-92, 2011 .
12. Bartel DP: MicroRNAs: Target recognition and regulatory functions. Cell 136: 215-233, 2009

13. Bhattacharyya SN, Habermacher R, Martine U, Closs EI and Filipowicz W: Stress-induced reversal of microRNA repression and mRNA P-body localization in human cells. Cold Spring Harb Symp Quant Biol 71: 513-521, 2006.
14. Li R, Li X, Ning S, Ye J, Han L, Kang C and Li X: Identification of a core miRNA-pathway regulatory network in glioma by therapeutically targeting miR-181d, miR-21, miR-23b, $\beta$-Catenin, CBP, and STAT3. PLoS One 9: el01903, 2014.

15. Wang L, ShiZM, Jiang CF, Liu X, Chen QD, Qian X, Li DM, Ge X, Wang XF, Liu LZ, et al: MiR-143 acts as a tumor suppressor by targeting N-RAS and enhances temozolomide-induced apoptosis in glioma. Oncotarget 5: 5416-5427, 2014.

16. Rathod SS, Rani SB, Khan M, Muzumdar D and Shiras A: Tumor suppressive miR-34a suppresses cell proliferation and tumor growth of glioma stem cells by targeting Akt and Wnt signaling pathways. FEBS Open Bio 4: 485-449, 2014.

17. Chang C, Shi H, Wang C, Wang J, Geng N, Jiang X and Wang X: Correlation of microRNA-375 downregulation with unfavorable clinical outcome of patients with glioma. Neurosci Lett 531: 204-208, 2012.

18. Shan B, Gerez J, Haedo M, Fuertes M, Theodoropoulou M, Buchfelder M, Losa M, Stalla GK, Arzt E and Renner U: RSUME is implicated in HIF-1-induced VEGF-A production in pituitary tumour cells. Endocr Relat Cancer 19: 13-27, 2012.

19. Benderro GF, Sun X, Kuang $Y$ and Lamanna JC: Decreased VEGF expression and microvascular density, but increased HIF- 1 and $2 \alpha$ accumulation and EPO expression in chronic moderate hyperoxia in the mouse brain. Brain Res 1471: 46-55, 2012.

20. Li R, Wei J,Jiang C, Liu D, Deng L, Zhang K and Wang P: Akt SUMOylation regulates cell proliferation and tumorigenesis. Cancer Res 73: 5742-5753, 2013.

21. Capili AD and Lima CD: Structure and analysis of a complex between SUMO and Ubc9 illustrates features of a conserved E2-Ub1 interaction. J Mol Biol 369: 608-618, 2007.

22. Rapino C, Bianchi G, Di Giulio C, Centurione L, Cacchio M, Antonucci A and Cataldi A: HIF-1alpha cytoplasmic accumulation is associated with cell death in old rat cerebral cortex exposed to intermittent hypoxia. Aging Cell 4: 177-185, 2005.

23. Melchior F- SUMO-nonclassical ubiquitin. Annu Rev Cell Dev Biol 16: 591-626, 2000.

Vertegaal AC, Ogg SC, Jaffray E, Rodriguez MS, Hay RT, Andersen JS, Mann M and Lamond AI: A proteomic study of SUMO-2 target proteins. J Biol Chem 279: 33791-33798, 2004.

Wei R, Yang Q, Han B, Li Y, Yao K, Yang X, Chen Z, Yang S, Zhou J, Li M, et al: microRNA-375 inhibits colorectal cancer cells proliferation by downregulating JAK2/STAT3 and MAP3K8/ERK signaling pathways. Oncotarget 8: 16633-16641, 2017.

26. Tsukamoto Y, Nakada C, Noguchi T, Tanigawa M, Nguyen LT, Uchida T, Hijiya N, Matsuura K, Fujioka T, Seto M and Moriyama M: MicroRNA-375 is downregulated in gastric carcinomas and regulates cell survival by targeting PDK1 and 14-3-3zeta. Cancer Res 70: 2339-2349, 2010. 